\title{
Proof of Renormalizability of Sclar Field Theories Using the Epstein- Glaser Scheme and Techniques of Microlocal Analysis
}

\author{
Lucas T. Cardoso \\ Departamento de Física, Universidade Federal de Juiz de Fora \\ 36036-900 Juiz de Fora, MG, Brasil \\ Itcfisica@yahoo.com.br
}

\begin{abstract}
The renormalizability of QFT's is a vastly studied issue, and particularly the results concerning a scalar field theory are well-known through the traditional renormalization approach in the literature. However, in this paper we analyze the problem through a less known approach, which justifies in a more rigorous and mathematically neat manner, the heuristic arguments of standard treatments of divergencies in QFT's. This paper analyzes the renormalizability of an arbitrary scalar field theory with interaction Lagrangean $L(x)=: \varphi^{m}(x)$ : using the method of Epstein-Glaser and techniques of microlocal analysis, in particular, the concept of scaling degree of a distribution. For a renormalizability proof of perturbative models in the Epstein-Glaser scheme one first needs to define an $n$-fold product of sub-Wick monomials of the interaction Lagrangean. This time ordering is an operator-valued distribution on $\mathbb{R}^{4 n}$ and the basic issue is its illdefinedness on a null set. The renormalization of a theory in this scheme amounts to the problem of extension of distributions across null sets.
\end{abstract}

\section{Keywords}

Quantum Field Theory, Renormalization, Epstein-Glaser scheme, Microlocal analysis, scalar field theory, scaling degree, time-ordered product.

\section{Introduction}

The problem of infinities is already present in classical electromagnetism. It is a known fact that if one tries to calculate the electron self-energy due to its electric field considering the electron as a point-like particle, one is left with an integral which diverges as the radius of the electron goes to zero, i.e. $r \rightarrow 0$. Unfortunately ${ }^{1}$, as first noted by Heisenberg and Pauli $[4,6]$, the problem of infinities emerged even more drasticly in Quantum Field Theory. Analagously to the classical case, in Quantum Electrodynamics (QED) there is a problem of divergence that arises when one tries to calculate perturbatively the electron and photon self-energies, for instance. The Renormalization Theory is a process devised initially as a way to eliminate these infinities arising in QED and other QFT's [5, 7, 10, 9, 8, 13, 11, 12, 15, 14, 16, 17]. It states essentially that the parameters appearing in the Lagrangean of the theory have no direct physical interpretation, and one can adjust them in order to absorb the divergencies coming out in the perturbative calculations at each order in the perturbative expansion. A physical interpretation of this redefinition of parameters can be motivated by the following example: Suppose an electron is moving in the interior of a solid substance. Due to its interaction with the internal structure of the substance, the electron's effective mass $m^{*}$, which determines its response to the application of an external force, is certainly different from the electron's mass $m$ measured outside of the solid. The electron's mass is changed (renormalized) from $m$ to $m^{*}$ due to the interaction between the internal structure of the material and the electron. Along the same lines, it may be interpreted that the infinite electron's "self-mass" is produced by interaction of the electron with its own virtual photon cloud. The infinities that have to be renormalized in QFT come from the short distance, or equivalently large momenta, behaviour of the Feynman propagators, and are thus called ultraviolet divergencies.

From almost a century of careful research in QFT, a lot more has been completely understood about renormalizability. However, the main approach to renormalization is still through a redefinition of "infinite parameters" in the Lagrangean of the theory. Yet successful and perfectly understandable, a more rigorous way of dealing with the delicacy of the problem of divergencies in QFT has been highly desirable since its emergence. In the words of Dirac himself (1975) [18]: "Most physicists are very satisfied with the situation. They say: 'Quantum electrodynamics is a good theory and we do not have to worry about it any more.' I must say that I am very dissatisfied with the situation, because this so-called 'good theory' does involve neglecting infinities which appear in its equations, neglecting them in an arbitrary way. This is just not sensible mathematics. Sensible mathematics involves neglecting a quantity when it is small not neglecting it just because it is infinitely great and you do not want it!"

One important part of renormalization is the process of regularization, which roughly speaking consists in a method for handling divergent integrals by the introduction of a suitable parameter called regulator. The regularization essentially separates divergent integrals into finite and infinite parts, these last ones are ultimately absorbed in the redefinition of parameters with renormalization. Although, many regularization methods have been quite successful, there are ambiguities associated to the extracting of the finite part of a divergent integral [22, 23, 24], which is natural since even the more fundamental axioms of arithmetic are only applied to numbers. However, since every physical process in perturbative quantum field theory (PQFT) is expressed in terms of Feynman propagators, which are not functions but 
distributions, we may expect that the most resoanable means to treat Feynman amplitudes is by considering its distributional character and applying the powerful theory of distributions.

The first step towards a more rigorous treatment of PQFT is due to H. Epstein and V. J. Glaser [3]. The perturbative construction of interaction models a la Epstein and Glaser depends fundamentally on time ordered products of sub-Wick polynomials $W_{k}$ of the interaction Lagrangean $L$, denoted by $T_{n}\left(W_{1}, W_{2}, \ldots, W_{n}\right)$. These are operatorvalued distributions on $\mathbb{R}^{4 n}$ acting on the domain $\mathcal{D}$ of vectors with finite particle number and smooth momentum space wave functions. The $T$-product is a well-defined distribution outside the subset of $\mathbb{R}^{4 n}$ consisting of all coincidental points, called the total diagonal $\Delta_{n} \doteq\left\{\left(x_{1}, \ldots, x_{n}\right): x_{1}=\ldots=x_{n}\right\}$. The problem of renormalization in the Epstein-Glaser scheme corresponds to the extension of the $T$ - product across $\Delta_{n}$. This extension is, in general not unique, depending on the scaling degree ${ }^{2}$ of the distribution involved. However, the distribution is unique up to derivatives of delta distribution (see 4.9). Each derivative of the delta is multiplied by an arbitrary constant. The redefinition of the parameters in standard Renormalization theory corresponds to the fixing of these constants, which are realized by requiring the maintenance of physical principles such as conservation of energy and momentum. Having constructed the $T$-product, the Bogoliubov's S-matrix is defined as a functional associating with a test function $g \in \mathcal{D}\left(\mathbb{R}^{4}\right)$ and an interaction Lagrangean $L$ the following series

$$
\begin{aligned}
S(g L) & \doteq \sum_{n=0}^{\infty} \frac{i^{n}}{n !} T_{n}(L, L, \ldots, L)(g, g, \ldots, g) \\
& =\sum_{n=0}^{\infty} \frac{i^{n}}{n !} \int d^{4} x_{1} \cdots d^{4} x_{n} g\left(x_{1}\right) \cdots g\left(x_{n}\right) T_{n}\left(L\left(x_{1}\right), \cdots, L\left(x_{n}\right)\right),
\end{aligned}
$$

in the formal sense, i.e. without requiring the convergence of the series. In the so called adiabatic limit, $g(x) \rightarrow g=$ constant, equation (1.1) becomes the S-matrix of the model.

A very general treatment of Renormalization of QFT's using the method of Epstein-Glaser and techniques of microlocal analysis is exposed in a monumental work by K. Fredenhagen and R. Brunetti [1]. However, in this work, we give a simpler and more detailed analysis restricted to the four dimensional Minkowski spacetime. In particular, we prove the renormalizability of an arbitrary scalar field theory with no derivatives and interaction Lagrangean $L(x)=: \varphi^{m}(x)$ : through the calculation of the scaling degree of the distribution associated to an arbitrary $n$-fold time-ordered product of sub-Wick monomials of the interaction lagrangean.

\footnotetext{
${ }^{1}$ Or fortunately, if we consider the vast amount of development in mathematics and in the fundamentals of physics that sprang from the paradigm of infinities in Quantum Field Theory.

${ }^{2}$ For the definition of scaling degree see 4.3
} 
2 Renormalizability of $L(x)=: \varphi^{m}(x)$ :

Let $\varphi$ be a point-like operator-valued field, then the time-ordering of the product $\varphi\left(x^{\prime}\right) \varphi(x)$ is given by ${ }^{3}$

$$
T \varphi(x) \varphi\left(x^{\prime}\right)=\left\{\begin{array}{l}
\varphi(x) \varphi\left(x^{\prime}\right) \text { if } x \succcurlyeq x^{\prime} \\
\varphi\left(x^{\prime}\right) \varphi(x) \text { if } x^{\prime} \succcurlyeq x
\end{array} .\right.
$$

where $x \succcurlyeq y$ means, by definition, that $x$ is not contained in the past light-cone of $y$, i.e. $x \succcurlyeq y: \Leftrightarrow x \notin \overline{V_{-}(y)}$. We may read the symbol " $\succcurlyeq$ " as "later than". Furthermore, if the points $x$ and $y$ are space-like separated, then both $x \succcurlyeq y$ and $y \succcurlyeq x$ hold, but even so, by the causality axiom, which asserts the commutativity of fields for space-like separated events, there is no ambiguity to equation (2.1), hence equation (2.1) is well-defined for every pair of points $\left(x, x^{\prime}\right) \in \mathbb{R}^{8} \backslash \Delta_{2}$, where $\Delta_{2} \doteq\left\{\left(x, x^{\prime}\right) \in \mathbb{R}^{8}: x=x^{\prime}\right\}$. Thus, the $T$-product is Lorentz invariant and hence well-defined for noncoincidental points.

Using Wick's expansion theorem, we may rewrite (2.1) as

$$
T \varphi(x) \varphi\left(x^{\prime}\right)=: \varphi(x) \varphi\left(x^{\prime}\right):+\left(\Omega, T \varphi(x) \varphi\left(x^{\prime}\right) \Omega\right),
$$

where the double colon stands for the normal order relation and $\left(\Omega, T \varphi(x) \varphi\left(x^{\prime}\right) \Omega\right):=\Delta_{F}\left(x-x^{\prime}\right)$ is the Feynman propagator associated with $\varphi$. The case in which $x$ coincides with $x^{\prime}$ is reasonably treated by extending the numerical distribution given by the Feynman propagator in equation (2.2) across the diagonal $\Delta_{2} \doteq\left\{\left(x, x^{\prime}\right) \in \mathbb{R}^{8}: x=x^{\prime}\right\}$. This last step is where the subtleties actually lie.

In order to study the time ordering of an arbitrary product of sub-Wick monomials of the interaction lagrangean one needs to know its fundamental properties. Let us first start with the following informal definition

$$
T_{n}\left(W_{1}, \ldots, W_{n}\right)\left(f_{1}, \ldots, f_{n}\right)=: \int_{\left(\mathbb{R}^{4}\right)^{\times n}} d x_{1} \cdots d x_{n} T_{n}\left(W_{1}\left(x_{1}\right) \cdots W\left(x_{n}\right)\right) f_{1}\left(x_{1}\right) \cdots f_{n}\left(x_{n}\right),
$$

where $f_{1}, \cdots f_{n} \in \mathcal{D}\left(\mathbb{R}^{4}\right)$ and $T_{0}$ and $T_{1}$ are given by $T_{0}:=1$ and $T_{1}(W):=W$. Further requirements are:

i. (Linearity.) The time-ordered product $T_{n}$ is an $n$-linear application from the Wick polynomials into operator-valued distributions acting on $\mathcal{D}^{5}$.

ii. (Symmetry.) $T^{n}\left(W_{1}, \ldots, W_{n}\right)$ is symmetric under permutations $W_{i} \leftrightarrow W_{k}$.

iii. (Causality.) If $x_{i} \succcurlyeq x_{j}$ for all $i \in\{1, \ldots, k\}$ and $j \in\{k+1, \ldots, n\}$, then the following factorization holds:

$$
T_{n}\left(W_{1}\left(x_{1}\right) \cdots W_{n}\left(x_{n}\right)\right)=T_{k}\left(W_{1}\left(x_{1}\right) \cdots W_{k}\left(x_{k}\right)\right) T_{n-k}\left(W_{k+1}\left(x_{k+1}\right) \cdots W_{n}\left(x_{n}\right)\right) .
$$

iv. (Covariance.) If $W_{i}$ are scalar Wick polynomials, then for all $(a, \Lambda) \in P_{+}^{\uparrow}$

$$
U(a, \Lambda) T_{n}\left(W_{1}\left(x_{1}\right) \cdots W_{n}\left(x_{n}\right)\right) U(a, \Lambda)^{-1}=T_{n}\left(W_{1}\left(a+\Lambda x_{1}\right) \cdots W_{n}\left(a+\Lambda x_{n}\right)\right) .
$$

v. (Wick expansion - scalar field case.) Let $k_{1}, \ldots, k_{n} \in \mathbb{N}_{0}$ and let $\mathcal{G}\left(k_{1}, \ldots, k_{n}\right) \doteq\left\{0, \ldots, k_{1}\right\} \times \cdots \times\left\{0, \ldots, k_{n}\right\}$ be the set of multi-indices $\left(l_{1}, \ldots, l_{n}\right) \in \mathbb{N}_{0}^{\times n}$ with $0 \leq l_{i} \leq k_{i}$. Then

$$
T_{n}\left(: \varphi^{k_{1}}\left(x_{1}\right): \cdots: \varphi^{k_{n}}\left(x_{n}\right):\right)=\sum_{G \in \mathcal{G}\left(k_{1}, \ldots, k_{n}\right)} t_{G}\left(x_{1}, \ldots, x_{n}\right): \varphi^{l_{1}}\left(x_{1}\right) \cdots \varphi^{l_{n}}\left(x_{n}\right):,
$$

where for $G=\left(l_{1}, \ldots, l_{n}\right), t_{G}$ is the numerical distribution

$$
t_{G}\left(x_{1}, \ldots, x_{n}\right)=\left(\begin{array}{c}
k_{1} \\
l_{1}
\end{array}\right) \cdots\left(\begin{array}{c}
k_{n} \\
l_{n}
\end{array}\right)\left(\Omega, T_{n}: \varphi^{k_{1}-l_{1}}\left(x_{1}\right): \cdots: \varphi^{k_{n}-l_{n}}\left(x_{n}\right): \Omega\right) .
$$


(The product of distributions in Eq. (2.3) exists due to Epstein-Glaser's "Theorem 0"[3].) The time-ordered products can be constructed inductively: If all $T_{k}$ are known up to some order $k \leq n-1$, then $T_{n}$ is fixed [3, 1] by causality and symmetry outside the small diagonal $\Delta_{n}$. Let us prove this claim as a theorem:

Theorem 2.1 Let $L$ be a particular functional lagrangean and denote by $T_{n}\left(x_{1}, \ldots, x_{n}\right) \equiv T L\left(x_{1}\right) \cdots L\left(x_{n}\right), n \in \mathbb{N}$, the family of symmetric operator-valued distributions on $\mathbb{R}^{4 n}$ satisfying the causality property. Then equation

$$
T_{n}\left(x_{1}, \ldots, x_{n}\right)=T_{k}\left(x_{1}, \ldots, x_{k}\right) T_{n-k}\left(x_{k+1}, \ldots, x_{n}\right) \text {. }
$$

fixes $T_{n}$ inductively up to the total diagonal $\Delta_{n} \doteq\left\{\left(x_{1}, \ldots, x_{n}\right): x_{1}=\ldots=x_{n}\right\}$ in the following sense: If $T_{k}$ is known for every $k<n$, then $T_{n}$ is fixed on $\mathbb{R}^{4 n} \backslash \Delta_{n}$.

Proof. Suppose $\left(x_{1}, \ldots, x_{n}\right) \notin \Delta_{n}$, then there are at least two points in the set $\left\{x_{1}, x_{2}, \ldots, x_{n}\right\}$ and hence there exists a partition $P=\left\{I, I^{c}\right\}$ of the set $\{1, \ldots, n\}$ such that $x_{i} \succeq x_{i^{\prime}} \forall i \in I$ and $\forall i^{\prime} \in I^{c}$. Thus, the causality property of the $T$-product implies

$$
T_{n}(\{1, \ldots, n\})=T(I) T\left(I^{c}\right)
$$

where $T(I):=T\left(x_{i_{1}}, \ldots, x_{i_{k}}\right), I=\left\{i_{1}, \ldots, i_{k}\right\}$ and $k=|I|$. If the points $x_{i}$ are all causally connected, that is $x_{i}^{2} \geq 0$ $\forall i \in\{1, \ldots, n\}$, then $I$ is uniquely defined $\left(x_{i_{1}} \succeq x_{i_{2}} \succeq \cdots \succeq x_{i_{n}}\right.$ for all reference frames, where $\left.\left\{i_{1}, \ldots, i_{n}\right\}=\{1, \ldots, n\}\right)$ and we are done. If, on the other hand, $\exists l \in\{1, \ldots, n\}$ such that $x_{l}^{2}<0$, (that is, $x_{l}$ is space-like) then $I$ is not uniquely defined, and in this case we must prove the independence of equation (2.5) on the choice of the proper subset $I$. With that purpose in mind, let $Q=\left\{J, J^{c}\right\}$ be another partition of the set $\{1, \ldots, n\}$ such that $x_{j} \succeq x_{j^{\prime}} \forall j \in J$ and $\forall j^{\prime} \in J^{c}$ Using the identities $I=I \cap J \cup I \cap J^{c}$ and $I^{c}=I^{c} \cap J \cup I^{c} \cap J^{c}$, we can rewrite the right-hand side of equation (2.5) as

$$
T(I) T\left(I^{c}\right)=T\left(I \cap J \cup I \cap J^{c}\right) T\left(I^{c} \cap J \cup I^{c} \cap J^{c}\right) .
$$

It is easy to see that $I \cap J \succeq I \cap J^{c}$ and $I^{c} \cap J \succeq I^{c} \cap J^{c}$ ( in the sense of the elements of the corresponding sets) and so (2.6) becomes

$$
T(I) T\left(I^{c}\right)=T(I \cap J) T\left(I \cap J^{c}\right) T\left(I^{c} \cap J\right) T\left(I^{c} \cap J^{c}\right)
$$

Analagously, for the sets $J$ and $J^{c}$ we have the following identities $J=J \cap I \cup J \cap I^{c} \quad$ and $J^{c}=J^{c} \cap I \cup J^{c} \cap I^{c}$, yield

$$
T(J) T\left(J^{c}\right)=T\left(J \cap I \cup J \cap I^{c}\right) T\left(J^{c} \cap I \cup J^{c} \cap I^{c}\right),
$$

and since both $J \cap I \succeq J \cap I^{c}$ and $J^{c} \cap I \succeq J^{c} \cap I^{c}$, we have

$$
T(J) T\left(J^{c}\right)=T(J \cap I) T\left(J \cap I^{c}\right) T\left(J^{c} \cap I\right) T\left(J^{c} \cap I^{c}\right) .
$$

It is clear that the arguments of the second and third terms in equation (2.9) are such that $J \cap I^{c} \succeq J^{c} \cap I$ from the perspective of the partition $Q$. However, from the perspective of the partition $P$ we also have $J^{c} \cap I \succeq J \cap I^{c}$. This means that the points in $J^{c} \cap I$ are causally disjoint from the points in $J \cap I^{c}$, which enables us to commute the terms $T\left(J \cap I^{c}\right)$ and $T\left(J^{c} \cap I\right)$ in (2.9) obtaining as a result 


$$
T(J) T\left(J^{c}\right)=T(J \cap I) T\left(J^{c} \cap I\right) T\left(J \cap I^{c}\right) T\left(J^{c} \cap I^{c}\right)=T(I) T\left(I^{c}\right)
$$

From the previous theorem we know that the time-ordering of an $n$-fold product of any point-like interaction lagrangean $L(x)$ is well-defined everywhere, except in the total (small) diagonal $\Delta_{n}$.

The "UV problem" of divergencies consists, in this context, in the extension of the $T$-product across $\Delta_{n}$. This extension is not unique in general, and the choice of possible extensions is restricted by the requirements of covariance and Wick expansion for the $T$-product, which therefore may be called (re-) normalization conditions. By covariance, the $T_{n}$ are fixed up to the origin in $\mathbb{R}^{4 n}$. The condition of Wick expansion holds due to Wick's theorem outside the union of all diagonals, i.e., whenever $x_{i} \neq x_{j}$, and the requirement that it holds on all $\mathbb{R}^{4 n}$ is a normalization condition by virtue of which the extension problem needs to be considered only for the numerical distributions $t_{G}$.

Let us now investigate the renormalizability of a scalar theory whose interaction lagrangean is of the form $L(x)=: \varphi^{m}(x):$. In this case, the Wick expansion (2.3) for $n$ vertices is given by

$$
T L\left(x_{1}\right) \cdots L\left(x_{n}\right)=\sum_{G \in \mathcal{G}(m, \ldots, m)} t_{G}\left(x_{1}, \ldots, x_{n}\right): \varphi^{b_{1}}\left(x_{1}\right) \cdots \varphi^{b_{n}}\left(x_{n}\right):
$$

where for $G=\left(b_{1}, \ldots, b_{n}\right), t_{G}$ is the numerical distribution

$$
t_{G}\left(x_{1}, \ldots, x_{n}\right)=\left(\begin{array}{l}
m \\
b_{1}
\end{array}\right) \ldots\left(\begin{array}{l}
m \\
b_{n}
\end{array}\right)\left(\Omega, T_{n}: \varphi^{m-b_{1}}\left(x_{1}\right): \cdots: \varphi^{m-b_{n}}\left(x_{n}\right): \Omega\right) .
$$

Applying Wick's expansion to both sides of equation (2.5) we get

$$
\begin{aligned}
& \sum_{G \in \mathcal{G}_{n}} t_{G}\left(x_{1}, \cdots, x_{n}\right): \varphi^{b_{1}}\left(x_{1}\right) \cdots \varphi^{b_{n}}\left(x_{n}\right): \\
& =\sum_{G_{1} \in \mathcal{G}_{k}} \sum_{G_{2} \in \mathcal{G}_{n-k}} t_{G_{1}}(I) t_{G_{2}}\left(I^{c}\right): \varphi^{b_{i_{1}}}\left(x_{i_{1}}\right) \cdots \varphi^{b_{i_{k}}}\left(x_{i_{k}}\right):: \varphi^{b_{i_{k+1}}}\left(x_{i_{k+1}}\right) \cdots \varphi^{b_{i_{n}}}\left(x_{i_{n}}\right): \\
& =\sum_{G_{1}, G_{2}} t_{G_{1}}(I) t_{G_{2}}\left(I^{c}\right): \varphi^{b_{I}}(I):: \varphi^{{ }^{I^{c}}}\left(I^{c}\right):
\end{aligned}
$$

where $\mathcal{G}_{n}$ is the set of graphs with $n$ vertices $x_{1}, \cdots, x_{n}$ and with $b_{i}$ external lines coming out of the vertex $x_{i}$. The set $\left\{I, I^{c}\right\}$ is a partition of the set $\left\{x_{1}, \cdots, x_{n}\right\}$ with $I=\left\{x_{i_{1}}, \cdots, x_{i_{k}}\right\}$ and $I^{c}=\left\{x_{1}, \cdots, x_{n}\right\} \backslash I=\left\{x_{i_{k+1}}, \cdots, x_{i_{n}}\right\}$. Also, we have defined $\varphi^{b_{i_{1}}}\left(x_{i_{1}}\right) \cdots \varphi^{b_{i_{k}}}\left(x_{i_{k}}\right) \stackrel{\text { def }}{=} \varphi^{b_{I}}(I)$, where $b_{I}=b_{i_{1}}+\cdots+b_{i_{k}}$ and $b_{I^{c}}=b_{i_{k+1}}+\cdots b_{i_{n}}$ are the total number of external lines coming out of the $I$ and $I^{c}$ vertices, respectively, in the graph $G$.

Using Wick's expansion for the product of the normal ordered terms in equation (2.13) we get

$$
\begin{aligned}
& \sum_{G} t_{G}\left(x_{1}, \cdots, x_{n}\right): \varphi^{b_{1}}\left(x_{1}\right) \cdots \varphi^{b_{n}}\left(x_{n}\right): \\
& =\sum_{G_{0}, G_{1}, G_{2}} t_{G_{1}}(I) t_{G_{2}}\left(I^{c}\right) \prod_{(i, j) \in I \times I^{c}}\left[\Delta_{F}\left(x_{i}-x_{j}\right)\right]^{l_{i j}}: \varphi^{b_{I}^{\prime}}(I) \varphi^{b^{\prime} I^{c}}\left(I^{c}\right):
\end{aligned}
$$


where $G_{0}$ runs through the graphs whose internal lines connect only vertices in $I$ with vertices in $I^{c}$, and $b:=b_{I}+b_{I^{c}}=b_{I}^{\prime}+b_{I^{c}}^{\prime}-2 l_{I, I^{c}}$, where $l_{I, I^{c}}=\sum l_{i j}$ is the number of lines connecting vertices in $I$ with vertices in $I^{c}$, as ilustrated in figures 1 and 2 . The term $\Delta_{F}$ represents the Feynman propagator ${ }^{4}$ given by equation (4.7) in appendix. We may now calculate the scaling degree (definition 4.3 in appendix 4 ) of the distribution $t_{G}$ by induction:

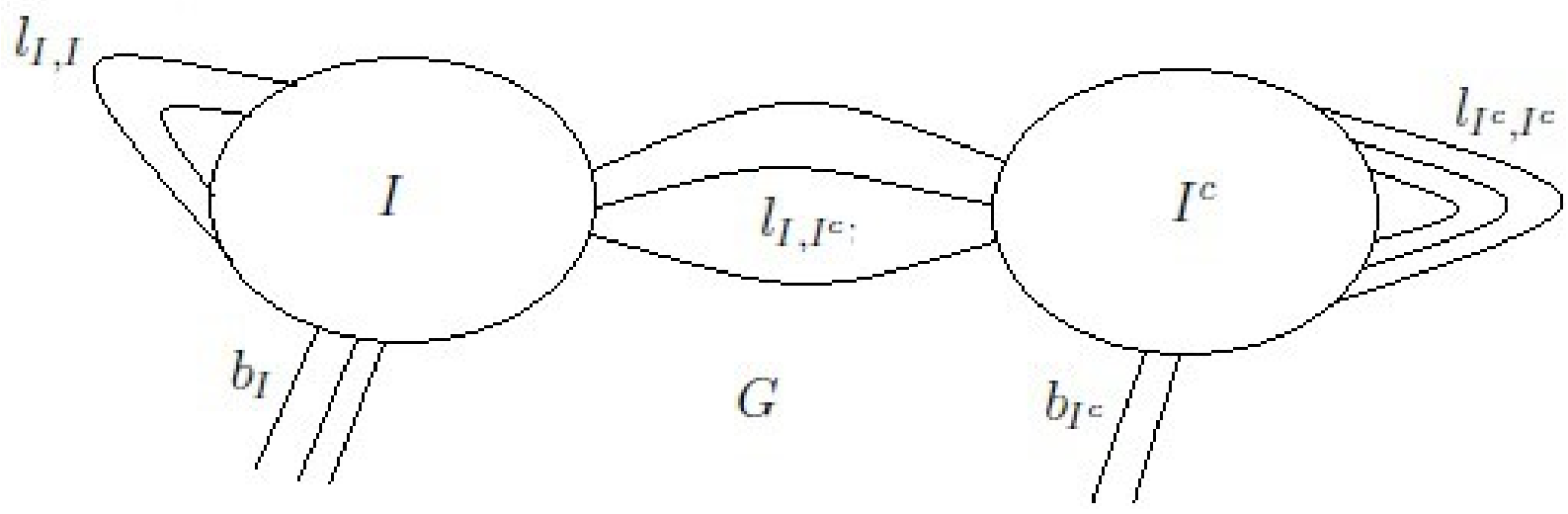

Figure 1: Graph $G$ with $n$ vertices $\left\{x_{1}, \cdots, x_{n}\right\}=I \cup I^{c}$ and $b_{I}+b_{I^{c}}$ external lines.

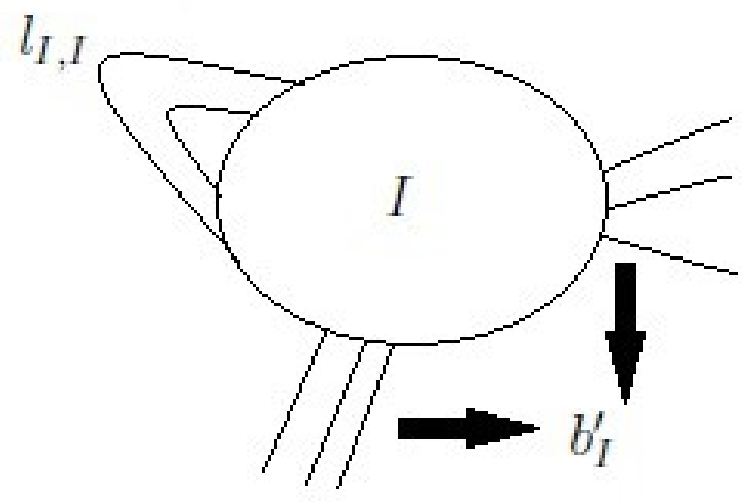

$G_{1}$

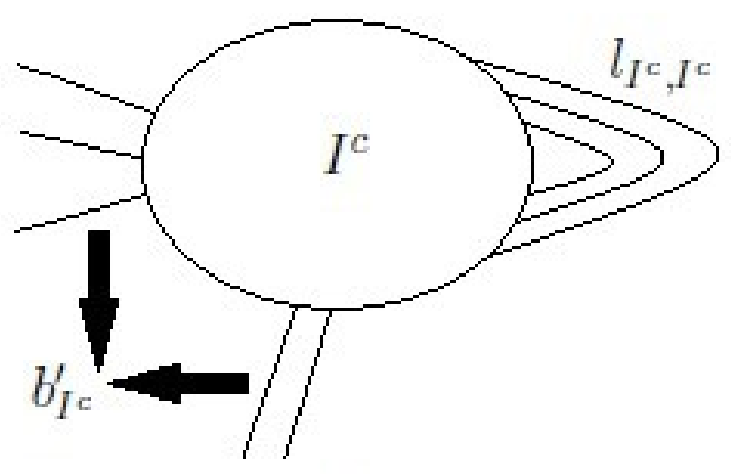

$G_{2}$

Figure 2: Dichotomy of the graph $G$ into $G_{1}$ and $G_{2}$, where graph $G_{1}$ has $k=|I|$ vertices and $b_{I}^{\prime}$ external lines and graph $G_{2}$ has $n-k=\left|I^{c}\right|$ vertices and $b_{I^{c}}^{\prime}$ external lines.

Theorem 2.2 For $G \in \mathcal{G}_{n}^{b}$, that is, $G$ has $n$ vertices and $b$ external lines, the distribution $t_{G}$ defined in (2.12) has scaling degree $m n-b$.

Proof. From equation (2.14) we get

$$
\operatorname{sd}\left(t_{G}\right)=\operatorname{sd}\left(t_{G_{1}}\right)+\operatorname{sd}\left(t_{G_{2}}\right)+\operatorname{sd}\left(\Delta_{F}\right) \sum l_{i j}
$$

where we have used the properties of scaling degree stated in 4.8. The graph $G_{1}$ has $k$ vertices and $b_{I}^{\prime}$ external lines whilst $G_{2}$ has $n-k$ vertices and $b_{I^{c}}^{\prime}$ external lines. Hence, using the induction hypothesis for $t_{G_{1}}$ and $t_{G_{2}}$ and the result $s d\left(\Delta_{F}\right)=2$ (obtained in example 4.7 in appendix 4), we get 


$$
\begin{aligned}
& \operatorname{sd}\left(t_{G}\right)=\left[m k-b_{I}^{\prime}\right]+\left[m(n-k)-b_{I^{c}}^{\prime}\right]+2 l_{I, I^{c}} \\
& =m n-\left(b_{I}^{\prime}+b_{I^{c}}^{\prime}\right)+2 l_{I, I^{c}} \\
& =m n-b
\end{aligned}
$$

To prove the basis step, note that for $n=1$ we have $t_{G}\left(x_{1}\right)=1$ with scaling degree 0 and $b=m$, hence $\operatorname{sd}\left(t_{G}\right)=0=m-b$. This completes the proof.

We may now calculate the degree of divergence ${ }^{6}$ of the distribution $t_{G}$ by

$$
\operatorname{div}\left(t_{G}\right):=\omega=\operatorname{sd}\left(t_{G}\right)-\operatorname{codim}\left(\Delta_{n}\right)
$$

where $\operatorname{codim}\left(\Delta_{n}\right)=\operatorname{dim}\left(\mathbb{R}^{4 n}\right)-\operatorname{dim}\left(\Delta_{n}\right)=4(n-1)$. Therefore,

$$
\omega=(m-4) n+4-b
$$

We may now analyze the renormalizability of a scalar theory with interaction lagrangean of the form $L(x)=: \varphi^{m}(x):$. By inspecting equation (2.20) we notice that if $m>4$, the degree of divergence will increase with the number of vertices no matter how large the number of external lines is, which makes the theory non-renormalizable in this case. As an example, for $m=4$ we have $\omega=4-b$ and only graphs with $n=2$ and $n=4$ will be superficially divergent, those with $0 \leq b \leq 4$. Therefore, the theory is renormalizable, since only a finite number of physical constants has to be redefined. The redefinition of physical parameters in standard renormalization schemes corresponds to the non-unique extension of distributions in the Epstein-Glaser scheme. As stated in theorem 4.9 in appendix 4, for $\omega \geq 0$ the extension of the distribution through the origin is unique up to derivatives of the delta distribution and each term contains an undefined constant which can be fixed by the imposition of physical principles, such as conservation of energy and momentum. The larger $\omega$ is, the larger will be the number of parameters to be fixed. Furthermore, if $\omega<0$ the extension is unique.

\footnotetext{
${ }^{3}$ We consider the case where the field $\varphi$ is bosonic. In the case it were fermionic, the second line in equation (2.2) would be $-\varphi(x) \varphi\left(x^{\prime}\right)$

${ }^{4}$ For the explicit form of the Feynman propagator for scalar fields see 4.7.

${ }^{5}$ We adopt here the "on-shell formalism".

${ }^{6}$ See the definition of degree of divergence in the last paragraph of appendix 4
}

\section{Final comments}

In this work, we discussed the renormalizability of a scalar field theory $L(x)=: \varphi^{m}(x)$ :, which is a well-known fact in the traditional literature in QFT. However, whilst the traditional approach is realized in a more intuitive and less rigorous way, we have used the more mathematically rigorous approach of Epstein-Glaser with a slight modification extensively treated by $\mathrm{K}$. Fredenhagen et al, which uses techniques of microlocal analysis for treating the extension problem, instead of the more arduous and elaborate technique of splitting of distributions.

We have constructed the time-ordered products of sub-Wick monomials of the interaction Lagrangean. Those $T$-products were proved by induction in theorem 2.1 to be well-defined distributions outside the meager set of all coincidental points in $\left(\mathbb{R}^{4}\right)^{\times n}$, which is a null subset $\Delta_{n}$. Later we classified the degree of freedom of the general $T$. product by calculating its scaling degree, which depended on the power of the field $m$ in the interaction, the number of vertices $n$, and the total number of external lines in the corresponding graph $G$ associated to the distribution $t_{G}$. Finally, the degree of divergence of the theory was shown to be $\omega=(m-4) n+4-b$, which emphasizes precisely the nonrenormalizability of a scalar field theory with power $m>4$ in the interaction Lagrangean, since in this case the degree of 
divergence will inevitably increase with the number of vertices of a grapgh $G$, independently on how large the number of external lines is.

The Epstein-Glaser approach is very fruitful and has been gaining the attention of physicists even from outside the area of mathematical and theoretical physics. More recently, the method has been successfully implemented in the relatively new approach for QFT's using string-localized fields ${ }^{7}$ instead of the usual point-localized ones [26].

${ }^{7}$ For the main work on this new approach see [25].

\section{Basic Notions on the Extension of Distributions and Scaling Degree}

As mentioned previously, the problem of ultraviolet divergence can be solved by the extension of certain distributions which in turn will account for the renormalization of the theory. In order to that one uses the notion of scaling degree of a distribution. In this section ${ }^{8}$, we shall introduce some properties of the scaling degree and provide some simple examples. Let us start with the following definition:

Definition 4.1 Let $\varphi \in \mathcal{D}\left(\mathbb{R}^{n}\right)$ and $\lambda \in \mathbb{R}_{+}$, then we define a dilataion $\Lambda$ of the function $\varphi$ through $\lambda$ by

$$
\begin{aligned}
& \Lambda: \mathbb{R}_{+} \times \mathcal{D}\left(\mathbb{R}^{n}\right) \rightarrow \mathcal{D}\left(\mathbb{R}^{n}\right) \\
& (\lambda, \varphi) \mapsto \varphi^{\lambda} \doteq \lambda^{-n} \varphi\left(\lambda^{-1} \cdot\right)
\end{aligned}
$$

By pullback ${ }^{9}$, we can define a dilatation of a distribution $u \in \mathcal{D}^{\prime}\left(\mathbb{R}^{n}\right)$ as

$$
\left(\Lambda^{*} u\right)(\varphi) \doteq u_{\lambda}(\varphi) \doteq u\left(\varphi^{\lambda}\right)
$$

For the case of $u \in L_{l o c}^{1}\left(\mathbb{R}^{n}\right)$ we can write equation (4.2) as the integral

$$
u_{\lambda}(\varphi)=\int u(\lambda x) \varphi(x) d^{n} x, \quad \forall \varphi \in \mathcal{D}\left(\mathbb{R}^{n}\right)
$$

The quantity $u(x)$ is referred to as the integral kernel of $u$ and we shall, by the usual abuse of notation, denote a general distribution $u(\varphi)$ as the integral in (4.3).

Let $\mathcal{D}\left(\mathbb{R}^{n} \backslash\{0\}\right)=\left\{\varphi \in \mathcal{D}\left(\mathbb{R}^{n}\right): 0 \notin \operatorname{supp}(\varphi)\right\}$ be the subspace of test functions whose support does not contain the origin and $\mathcal{D}^{\prime}\left(\mathbb{R}^{n} \backslash\{0\}\right)$ its dual ${ }^{10}$. With all that said, the central problem can be stated as follows:

Problem 4.2 Given a distribution $u_{0} \in \mathcal{D}^{\prime}\left(\mathbb{R}^{n} \backslash\{0\}\right)$, how can we construct a distribution $u \in \mathcal{D}^{\prime}\left(\mathbb{R}^{n}\right)$ such that $u_{0}(\varphi)=u(\varphi) \quad \forall \varphi \in \mathcal{D}\left(\mathbb{R}^{n} \backslash\{0\}\right) ?$

To answer that question one uses the concept of the so called scaling degree of a distribution, which basicly measures how singular a distribution is at the origin.

Definition 4.3 A distribution $u \in \mathcal{D}^{\prime}\left(\mathbb{R}^{n}\right)$ has scaling degree $s$ (in symbols $\operatorname{sd}(u)=s$ ) with respect to the origin in $\mathbb{R}^{n}$ if

$$
s \doteq \inf \left\{s^{\prime} \in \mathbb{R}: \lambda^{s^{\prime}} u_{\lambda} \stackrel{\lambda \rightarrow 0}{\longrightarrow} 0\right\}
$$

Let us see some simple examples.

Example 4.4 Let $f \in C^{0}(\mathbb{R})$ with $f(0) \neq 0$, then 


$$
\lim _{\lambda \downarrow 0} \lambda^{\omega} \int f(\lambda x) \varphi(x) d x= \begin{cases}0, & \text { if } \omega>0 \\ f(0) \int \varphi(x) d x, & \text { if } \omega=0 \\ \infty, & \text { if } \omega>0\end{cases}
$$

and consequently, $\operatorname{sd}(f)=0$.

Example 4.5 Let $\delta \in \mathcal{D}^{\prime}\left(\mathbb{R}^{n}\right)$ be the Dirac $\delta$-function. Since $\delta(\lambda x)=\lambda^{-n} \delta(x)$, we have $\operatorname{sd}(\delta)=n$.

Example 4.6 The functions $f(x)=e^{-\frac{1}{x^{2}}}$ and $g(x)=e^{\frac{1}{x}}$ both define distributions on $\mathbb{R} \backslash\{0\}$. Thus,

$$
\begin{cases}\lim _{\lambda \downarrow 0} \lambda^{s} f(\lambda x)=0 & \forall s \in \mathbb{R} \Leftrightarrow \operatorname{sd}(f)=-\infty \\ \lim _{\lambda \downarrow 0} \lambda^{s} g(\lambda x)=\infty & \forall s \in \mathbb{R} \Leftrightarrow \operatorname{sd}(g)=\infty\end{cases}
$$

Example 4.7 Consider the scalar Feynman propagator $\Delta_{F}$ of a massive scalar theory in four dimensions given by

$$
\Delta_{F}(x, m)=\lim _{\varepsilon \downarrow 0}(2 \pi)^{-4} \int d^{4} p \frac{e^{-i p \cdot x}}{\left(p^{2}-m^{2}+i \varepsilon\right)},
$$

then

$$
\begin{aligned}
\Delta_{F}(\lambda x, m) & =\lim _{\varepsilon \downarrow 0}(2 \pi)^{-4} \int d^{4} p \frac{e^{-i p \cdot \lambda x}}{\left(p^{2}-m^{2}+i \varepsilon\right)} \\
& =\lim _{\varepsilon \downarrow 0}(2 \pi)^{-4} \lambda^{-2} \int d^{4} p \frac{e^{-i p \cdot x}}{\left(p^{2}-(\lambda m)^{2}+i \varepsilon\right)} \\
& =\lambda^{-2} \Delta_{F}(x, \lambda m) .
\end{aligned}
$$

Since for $m \rightarrow 0, \Delta_{F}(x, m)$ converges to the massless scalar propagator of the theory, we have $\operatorname{sd}\left(\Delta_{F}\right)=2$.

The following theorem highlights some important properties of the scaling degree. It will be presented without proof (see [1]).

Theorem 4.8 Let $t \in \mathcal{D}^{\prime}\left(\mathbb{R}^{n}\right)$ have $\operatorname{sd}(t)=s$ at 0 , then the scaling degree obeys the following properties:

$\cdot \operatorname{sd}\left(\partial^{\alpha} t\right) \leq s+|\alpha|$, where $\alpha \in \mathbb{N}^{n}$ is any multiindex,

$\cdot \operatorname{sd}\left(x^{\alpha} t\right) \leq s-|\alpha|$, where $\alpha \in \mathbb{N}^{n}$ is any multiindex,

$\cdot \operatorname{sd}(f t) \leq \operatorname{sd}(t)$, where $f \in \mathcal{E}^{n}\left(\mathbb{R}^{n}\right)$,

$\cdot \operatorname{sd}\left(t_{1} \otimes t_{2}\right)=\operatorname{sd}\left(t_{1}\right)+\operatorname{sd}\left(t_{2}\right)$, for $t_{i} \in \mathcal{D}^{\prime}\left(\mathbb{R}^{n_{i}}\right), i=1,2$.

A precise answer to problem of uniqueness in the extension of distributions is given by the following theorem, which will be presented without proof ${ }^{11}$.

Theorem 4.9 Let $u_{0} \in \mathcal{D}^{\prime}\left(\mathbb{R}^{n} \backslash\{0\}\right)^{12}$. 
1. If $\operatorname{sd}\left(u_{0}\right)<n$, then there exists a unique extension $u \in \mathcal{D}^{\prime}\left(\mathbb{R}^{n}\right)$ such that $\operatorname{sd}\left(u_{0}\right)=\operatorname{sd}(u)$.

2. If $n \leq \operatorname{sd}\left(u_{0}\right)<\infty$, then there exist several extensions $u \in \mathcal{D}^{\prime}\left(\mathbb{R}^{n}\right)$ such that $\operatorname{sd}\left(u_{0}\right)=\operatorname{sd}(u)$. Given a particular solution $u_{p}$, the most general solution reads

$$
u=u_{p}+\sum_{|\alpha| \leq \mathrm{s}\left(u_{0}\right)-n} c_{\alpha} \partial^{\alpha} \delta^{(n)}
$$

with arbitray constants $c_{\alpha} \in \mathbb{C}$.

3. $\operatorname{sd}\left(u_{0}\right)=\infty$ If , then there exists no extension $u \in \mathcal{D}^{\prime}\left(\mathbb{R}^{n}\right)$.

It is convinient to define the notion of degree of divergence of a distribution $u$, which is given by $\operatorname{div}(u) \doteq \operatorname{sd}(u)-n$. It is worth mentioning that the non-unique extension case is given by (4.9) due to the fact that the most general distribution supported at the origin is given by an arbitrary differential polynomial applied to the $\delta$ distribution, see example 3.1.2 in [21].

\footnotetext{
${ }^{8}$ For more detailed description of the problem of extension of distributions consult $[19,20,1]$.

${ }^{9}$ The pullback essencialy transfers the effect of an operation over the test functions to an operation over the distributions by a composition. That is, let $X$ and $Y$ be open sets, $\Lambda$ be the mapping $\Lambda: \mathbb{R}_{+} \times X \subseteq \mathbb{R}_{+} \times \mathcal{D}\left(\mathbb{R}^{n}\right) \rightarrow Y \subseteq \mathcal{D}\left(\mathbb{R}^{n}\right)$ and $u$ be the distribution $u: Y \subseteq \mathcal{D}\left(\mathbb{R}^{n}\right) \rightarrow \mathbb{C}$, then $u \circ \Lambda:=\Lambda^{*} u: \mathbb{R}_{+} \times \mathcal{D}\left(\mathbb{R}^{n}\right) \rightarrow \mathbb{C}$.

${ }^{10}$ The extension exists by Hahn-Banach theorem, see [21, Chap. 3.2].

${ }^{11}$ For a detailed proof see [1]

${ }^{12}$ In the case the extension is not through the origin, but through an arbitrary submanifold $D$ of $\mathbb{R}^{n}$ we must replace $n$ in this theorem by the codimension of the submanifold, which is defined as $\operatorname{codim}(D)=\operatorname{dim}\left(\mathbb{R}^{n}\right)-\operatorname{dim}(D)=n-\operatorname{dim}(D)$.
}

\section{Acknowledgments}

The author is immensely grateful to Carlos A. Hernaski for valuable comments and motivational discussions and to Professor Jens K. H. Mund for clarifying discussions and teachings on the subject. A special thanks goes to Silas Matos for typing the final version of the article. The author has received financial support by the Brazilian research agency CAPES.

\section{References}

[1] R. Brunetti and K. Fredenhagen, Microlocal analysis and interacting quantum field theories: Renormalization on physical backgrounds, Commun. Math. Phys. 208 (2000), 623-661.

[2] D. Buchholz and K. Fredenhagen, Locality and the structure of particle states, Commun. Math. Phys. 84 (1982), 1-54.

[3] H. Epstein and V. Glaser, The role of locality in perturbation theory, Ann. Inst. Henri Poincaré A 19 (1973), $211-295$.

[4] W. Heisenberg and W. Pauli, On Quantum Field Theory.(In German), Z. Phys. 56, 1 (1929).

[5] H. A. Bethe, The Electromagnetic shift of energy levels, Phys. Rev. 72, 339 (1947).

[6] W. Heisenberg and W. Pauli, On Quantum Field Theory. 2. (In German), Z. Phys. 59, 168 (1930).

[7] J. S. Schwinger, Quantum electrodynamics. III: The electromagnetic properties of the electron: Radiative corrections to scattering, Phys. Rev. 76, 790 (1949).

[8] J. S. Schwinger, Quantum electrodynamics. I A covariant formulation, Phys. Rev. 74, 1439 (1948).

[9] J. S. Schwinger, Quantum electrodynamics. 2. Vacuum polarization and selfenergy, Phys. Rev. 75, 651 (1948).

[10] J. S. Schwinger, On Quantum electrodynamics and the magnetic moment of the electron, Phys. Rev. 73,416 (1948).

[11] R. P. Feynman, A Relativistic cutoff for classical electrodynamics, Phys. Rev. 74, 939 (1948).

[12] R. P. Feynman, Space - time approach to quantum electrodynamics, Phys. Rev. 76, 769 (1949).

[13] R. P. Feynman, Relativistic cutoff for quantum electrodynamics, Phys. Rev. 74, 1430 (1948). 
[14] S. Tomonaga, On a relativistically invariant formulation of the quantum theory of wave fields, Prog. Theor. Phys. 1 , 27 (1946).

[15] Z. Koba, T. Tati and S. i. Tomonaga, On a Relativistically Invariant Formulation of the Quantum Theory of Wave Fields. II: Case of Interacting Electromagnetic and Electron Fields, Prog. Theor. Phys. 2, no. 3, 101 (1947).

[16] S. I. Tomonaga and J. R. Oppenheimer, On Infinite Field Reactions in Quantum Field Theory, Phys. Rev. 74, 224 (1948).

[17] F. J. Dyson, The Radiation theories of Tomonaga, Schwinger, and Feynman, Phys. Rev. 75, 486 (1949).

[18] H. Kragh, Dirac: A scientific biography, CUP 1990, ISBN: 0521380898, p. 184.

[19] M. Duetsch and K. Fredenhagen, A Local (perturbative) construction of observables in gauge theories: The Example of QED, Commun. Math. Phys. 203, 71 (1999).

[20] D. Prange, Epstein-Glaser renormalization and differential renormalization, J. Phys. A 32, 2225 (1999).

[21] L. Hörmander, The Analysis of Linear Partial Differential Operators I, Springer, 1983, Berlin.

[22] O. A. Battistel and G. Dallabona, From arbitrariness to ambiguities in the evaluation of perturbative physical amplitudes and their symmetry relations, Phys. Rev. D 65, 125017 (2002).

[23] E. Gambin, G. Dallabona and O. A. Battistel, Regularizations: A unique prescription for all situations, Braz. J. Phys. 37, 1191 (2007).

[24] O. A. Battistel and G. Dallabona, Consistency and universality in odd and even dimensional space-time QFT perturbative calculations," Int. J. Mod. Phys. A 29, no. 13, 1450068 (2014)

[25] J. Mund, B. Schroer and J. Yngvason, String localized quantum fields from Wigner representations, Phys. Lett. B 596, 156 (2004).

[26] L. T. Cardoso, J. Mund, and J. C. Varilly, String chopping and time-ordered products of linear string-localized quantum fields. Manuscript submitted to Adv. Math, Phys. on 09/06/2017. 